\title{
The role of the psycholinguistic characteristics of words in the assessment of language processing of patients with psychosis, ultra- high risk of psychosis, or schizophrenia
}

\author{
Vlasta Erdeljac • Martina Sekulić Sović • Draženka Ostojić2,3 • Aleksandar Savić2,4 \\ 'University of Zagreb, Faculty of Humanities and Social Sciences, Department of Linguistics, \\ 2University Psychiatric Hospital Vrapče, ${ }^{3}$ University of Zagreb, Faculty of Law, Social Work Study \\ Centre, ${ }^{4}$ University of Zagreb, School of Medicine, Chair of Psychiatry and Psychological Medicine
}

https://doi.org/10.17234/9789531758314.02

\section{Psychosis and schizophrenia-spectrum disorders}

Psychosis is an umbrella term used to describe disorders in which one's ability to adequately test reality is compromised because of changes in perception and thoughts as well as in affective responses, all of which leads to impaired functioning on multiple levels. The most common features in all psychotic disorders include delusions and hallucinations (often referred to as positive symptoms), but clinical presentation can vary significantly among individuals diagnosed with the same disorder, and even in the same individual over time, making the process of differential diagnosis in certain cases extremely complex.

Delusions are fixed false beliefs that resist change even when the individual is confronted with compelling contrary evidence; the most common are persecutory delusions (the belief that someone wants to harm or hurt the person, or is plotting against them) and referential delusions (the belief that random things/individuals have special meaning for the affected person). Other types of delusions can also be part of the clinical presentation (e.g. erotomanic, grandiose, somatic, and nihilistic delusions), and these can further be divided into bizarre and non-bizarre delusions.

Hallucinations are changes in perception that take place in the absence of any external stimulus and can affect any perceptual modality (visual, auditory, olfactory, gustatory, and somatic). Not only are delusions and hallucinations linked to 
psychosis, but other symptoms and symptom domains as well, such as disorganized thinking and speech, changes in motor behavior, and negative symptoms (e.g. flat affect, anhedonia, alogia, and amotivation). Certain symptom domains are seen more often in specific psychotic disorders (e.g. negative symptoms in schizophrenia), a fact that can be used in the process of reaching definitive diagnostic differentiation.

The disorder most commonly mentioned in the context of the psychosis spectrum is schizophrenia, which is often seen as the paradigmatic psychotic disorder. However, the spectrum also includes disorders such as schizoaffective disorder, schizophreniform disorder, delusional disorder, schizotypal disorder, and brief psychotic disorders. Psychosis symptoms can also be present in other psychiatric disorders (e.g. bipolar affective disorder, depression, and personality disorders) and can be induced by various psychoactive substances and medications or by various medical conditions (e.g. autoimmune, endocrine, and metabolic disorders, tumors, etc.).

A diagnosis of a psychotic disorder is primarily based on a detailed patient history and examination of mental status (whether by means of structured questionnaires or semi-structured and/or unstructured interviews). As of yet, there are no widely accepted "objective" neuroimaging or laboratory tests used to diagnose schizophrenia or other psychotic disorders (although such tests are used to diagnose medical conditions that potentially induce psychotic symptoms).

\section{Schizophrenia}

Schizophrenia (SCZ) is a psychotic disorder with a reported prevalence varying between $0.4 \%$ and $1 \%$ and is among those mental disorders with the greatest impact on the duration and quality of life as well as on the work and social functioning of those affected (Sadock, Sadock, \& Ruiz, 2015, p. 301; Bhugra, 2005; Mcgrath, Saha, Chant, \& Welham, 2008; Desai, Lawson, Barner, \& Rascati, 2013). The male to female rate ratio of SCZ is reported to be 1.4:1 (Desai et al., 2013), while the peak incidence in males is seen at an earlier age (15-25) than in females (25-35) (Sadock et al. 2015, p. 301; Mcgovern \& Cope, 1987). Women, however, show a bimodal distribution, and have a second peak of SCZ onset in middle age (Messias, Chen, \& Eaton, 2007). Men are reported to experience more negative symptoms, and the outcome for women is considered to be generally more favorable.

Although we referred in the previous paragraph to SCZ as a psychotic disorder, today it is considered to be a group of related disorders with possibly varying clinical presentations, underlying pathological mechanisms, and outcomes. One of the major, still unmet challenges in SCZ research today is therefore the identification of valid sub-types (both clinically and in relation to a number of different objective indicators). 


\subsection{Etiology}

Even though the etiology of SCZ has been studied since its initial conceptualization in the nineteenth century, no definitive model of its underlying pathology has emerged, which might at least partially be the result of its above-mentioned heterogeneity. We know from research carried out to date that there is a strong genetic component of the disorder, informing us of the risk to relatives of those affected, and recently, a number of additional candidate gene loci linked to SCZ have been identified (Schizophrenia Working Group, 2014), driving forward research on the genetics of the schizophrenia spectrum. The rate of concordance in monozygotic twins is reported to be between $33 \%$ and $50 \%$, and heritability in SZC spectrum disorders is 73\% (Sadock et al. 2015, p. 303; Hilker et al., 2018). Even if we take the higher rate of concordance (50\%) in monozygotic twins as true, it still indicates that SCZ vulnerability depends on more than just genetic loading.

The first studies on the neuropathology of SCZ failed to show clear signs of brain changes, causing the disorder to be classified as functional, but subsequent research indicated that there is a loss of brain volume, possibly stemming from excessive synaptic pruning during adolescence. In addition to changes observed in the prefrontal cortex, the limbic system, the thalamus, and some other specific regions of the brain, reduced symmetry was also reported. This is believed to originate during fetal life and to disrupt brain lateralization (Sadock et al. 2015, p. 304).

Probably the most widely accepted theory is the dopamine theory of SCZ (and psychosis in general), which argues that dysregulation of the dopamine system causes most symptoms of the disorder (particularly its positive and negative symptoms). This has been supported by the effectiveness of dopamine antagonists in the treatment of SCZ (for treating positive symptoms) and that of all of the currently available antipsychotic medications that regulate dopamine neurotransmission. Dopamine theory has survived many modifications, with changes in focus among different elements (dopamine production, release, receptor changes), and even after it was challenged by other hypotheses explaining the emergence of psychosis at different levels, it continues to be seen as the "final common pathway" to psychosis (Howes \& Kapur, 2009). The Glutamate and GABA systems dysregulation hypotheses postulate changes in excitation/inhibition, which have been confirmed by a number of studies and computer models of the disruptions seen in SCZ. Those changes have been linked to, among other things, working memory problems observed in the SCZ population (Starc et al., 2017). Neuroimaging studies of schizophrenia have also helped us to move from focusing on a specific brain region(s) to seeing it characterized by changes in widely distributed networks and functions (Yang et al., 2014). 
Many other theories on the etiology of SCZ and psychosis exist, possibly explaining other important elements of its underlying pathology (e.g. the infection hypothesis, based on, among other facts, a high incidence of SCZ after prenatal exposure to influenza), but listing all of them goes beyond the scope of this article. It is important to note, however, that there are also a number of psychological theories of SCZ (e.g. the double-bind concept) that have descriptive value and can help us understand individual symbolic meaning behind some of the symptoms, which helps in the treatment process when using psychological interventions for SCZ patients.

\subsection{Clinical presentation and diagnostic criteria}

No symptom is considered to be pathognomonic for schizophrenia, as all symptoms and signs reported in SCZ can also be seen in other psychiatric disorders. This, combined with the fact that we still do not have an objective diagnostic instrument, illustrates the difficulty in making an early diagnosis of SCZ and the importance of observations made over prolonged periods in order to capture all aspects of this psychopathology and its changes over time. Current conceptualizations of the schizophrenia spectrum recognize the existence of subtle, non-specific premorbid signs and symptoms progressing through the prodromal stage into frank psychosis. Patients who go on to develop schizophrenia-spectrum disorder may have been more introverted and socially retracted, and research has shown that cognitive changes may be among the first signs in those who go on to be diagnosed with SCZ (Häfner et al., 1992). Even with clear prodromal signs and symptoms predating the first episode of psychosis, we still consider prodromes to be a retrospective concept, clearly defined as part of SCZ only after the actual onset of the illness.

Keeping in mind that SCZ is a longitudinal concept without clear pathognomonic symptoms, we expect it to be present with a number of different signs and symptoms, changing over time, which we usually divide into different symptom domains. The clinical presentation of a patient with schizophrenia can range all the way from a disorganized, agitated, and bizarre patient to one who is seemingly inconspicuous, silent, and immobile. Two domains that have been part of all previous and current conceptualizations of psychosis and SCZ are positive and negative symptoms. Positive symptoms have occasionally additionally been divided into "core" positive symptoms reflecting perceptual disturbances and thought content (delusions and hallucinations), and formal thought disorder that reflects changes not in the content but rather in the organization of thoughts and speech. Disorders of the form of thought can be objectively assessed through a patient's speech and writing, and they include looseness of association, tangentiality, echolalia, verbigeration, derailment, and incoherence (with word salad 
being at its extreme point), as well as mutism. The thought process, on the other hand, can be inferred from a patient's behavior as well as speech or writing, and includes thought blocking and control, thought broadcasting, poverty of thought content, changes in abstract thinking, idiosyncratic associations, and perseveration.

Negative symptoms, which include flat affect, anhedonia, amotivation, and poverty of speech (alogia), usually raise less flags than positive symptoms early in the course of the disorder and are usually less prominent in other psychotic disorders than in SCZ. On the other hand, compared to some other symptoms, negative symptoms are linked to significant reductions in functioning and poor prognosis. In addition to these two symptom domains, we also identify mood changes, excitement, and the cognitive dimension. Symptoms in SCZ can be evaluated using unstructured, semi-structured, or structured interviews, and there are a number of questionnaires and scales which are used in research and clinical practice. One of the most widely used scales to assess the severity of symptoms is the Positive and Negative Syndrome Scale (PANSS), a 30-item scale in which each item is rated from 1 to 7 based on the severity of a specific phenomenon/symptom.

Changes in cognition in SCZ have been a particular focus of numerous studies and are now seen as central features of the disorder. Occasionally, cognition changes are even considered as candidates for trait markers of SCZ. Cognitive impairments precede the onset of psychosis and are relatively stable from the first episode of psychosis throughout the course of the disorder; in addition, they predict the level of social functioning (Häfner et al., 1992; Brewer et al., 2005). A number of studies of SCZ patients have found changes in attention, working memory, executive functioning, speed of processing, verbal comprehension, learning and memory, problem solving, and social cognition (Nuechterlein et al., 2004), with overall intelligence, executive functioning, and verbal memory being identified as especially influencing their social functioning (Green, Kern, Braff, \& Mintz, 2000; Leeson, Barnes, Hutton, Ron, \& Joyce, 2009). A wide array of cognitive impairments reflect the disruption of cortico-cerebellar-thalamic circuits, leading to what was termed "cognitive dysmetria" (Andreasen, Paradiso, \& Oleary, 1998).

Diagnosing SCZ is done according to accepted classification systems, two of the most widely currently accepted and used being the 10th revision of the International Statistical Classification of Diseases and Related Health Problems (ICD-10) (World Health Organization, 1992) and the 5th edition of the Diagnostic and Statistical Manual of Mental Disorders (DSM-5) (American Psychiatric Association, 2013). In essence, a person can be diagnosed if he/she meets a specified number of specific criteria with regard to symptoms, their duration, and reduction in functioning. According to DSM-5, in order for a diagnosis to 
be made, two symptoms from a list including delusions, hallucinations, disorganized speech (e.g. incoherence), disorganized behavior, and negative symptoms need to be present for most of the time during a one-month period (at least one of these two symptoms needs to be delusions, hallucinations, or disorganized speech). In addition to reduction of functioning, signs of the disturbance must also persist for at least six months (including the above-mentioned onemonth period). ICD-10, on the other hand, places more emphasis on specific symptoms, previously called first-rank symptoms. In order to be diagnosed with SCZ according to ICD-10, a person should have for most of the time during a one-month period one clear symptom from each of the following groups: (I) thought broadcasting, thought echo, thought insertion; (II) delusions of control, influence, or passivity; (III) voices commenting on the person's behavior or discussing that person among themselves; (IV) persistent delusions that are culturally inappropriate. The person can also be diagnosed if they have two less clear symptoms from two different above-mentioned groups, or symptoms from at least two other groups of symptoms (persistent hallucinations of any modality followed by delusions without affective content, changes in the train of thought resulting in incoherence or neologisms, catatonic behavior, negative symptoms, and significant changes in behavior and social functioning).

It is clear that the differences between these classification systems, whether we choose to see them as major or discreet, point to the problem of validity of the diagnosis of schizophrenia, which has implications in research but also clinical practice.

\section{First-episode psychosis (FEP)}

The past few decades have seen a rise in attempts at clarifying the complexity of SCZ as a heterogeneous syndrome and at identifying markers of its subtypes and predictors of different illness courses and outcomes. One of the most important "helper" concepts on that road is that of clinical staging, acting as a more refined way of diagnosing disorders across a timeline in hopes of allowing for early intervention at earlier stages of a disorder (Mcgorry et al., 2006). Staging rests on the idea of progressive reduction in functionality that comes with illness chronicity and assumes that early interventions might prove to be more effective and less harmful, and might be able to delay chronic phases. In order for staging to be meaningful, it is important that we achieve a broader knowledge of the etiology and course of a specific disorder, as well as of different risk and protective factors, markers and predictors, as well as their relationships, all of which seems to be mostly lacking in the case of schizophrenia and psychosis-spectrum disorders. It is thought that clinical staging might help in mapping various biological, cognitive, and other markers to a specific illness stage, thus also contributing 
to the validation of illness subtypes and possibly even separate clinical entities (Mcgorry, Killackey \& Yung, 2008).

When trying to formalize the stages of SCZ and define the extremes on the timeline, on one end of this line we usually position the chronic residual stage, and on the other the ultra-high-risk states, a concept that proves more difficult to define and operationalize. Authors have long talked about prepsychotic stages that precede frank psychosis, and we have already briefly mentioned the concept of prodromes earlier in this text. Prodromes include non-specific behavioral symptoms that can progress into subthreshold positive symptoms, sometimes years before actual symptoms of psychosis appear. Even with a number of initiatives trying to establish valid markers for states of clinical high risk for psychosis and predictors of transitioning into psychosis, which would help in defining certain symptoms as prodromal, we still talk about the prodromal phase as a retrospective concept, only after the onset of actual psychosis.

It might seem that, compared to prodromes, first-episode psychosis (FEP) must be something easier to define, as it rests on the appearance of the objectifiable actual psychotic symptoms that we listed earlier in this text. However, the clinical and research reality tends to deviate from that assumption. We can expect FEP to be conceptualized in terms of (i) first contact with psychiatric services and treatment (understandably an unreliable concept, given that symptoms can be present for years before treatment starts), (ii) duration of use of antipsychotics, and (iii) duration of psychosis (Breitborde, Srihari, \& Woods, 2009). In order to avoid pitfalls, the suggestion has been made that FEP should be re-conceptualized as early-course psychosis or recent-onset psychosis in terms of duration of psychosis, a definition that offers the promise of best construct validity. Given that most functional deterioration takes place in the first five years, the five-year point has often been used to mark the end of FEP or early-course psychosis, although one has to be mindful of significant individual variation in the course of the illness.

We are again using the umbrella term of psychosis, which reflects the fact that FEP can have various trajectories and outcomes, from differentiation into SCZ, affective psychosis, or other psychotic disorders, to full remission without subsequent episodes. ICD-10 recognizes variations in the clinical presentation of acute psychotic disorder (F23), describing them as polymorphic without SCZ symptoms (F23.0), polymorphic with SCZ symptoms (F23.1), schizophrenia-like (F23.2), predominantly delusional (F23.3), other specified (F23.8), or unspecified brief (reactive) (F23.9). There is little predictive value in these diagnoses, and so the diagnosis of schizophrenia-like acute FEP does not necessarily mean the person diagnosed with it will progress to actual SCZ. The variation of presentations, along with the fact that FEP can progress in the direction of affective psychosis, also means that patients in an acute psychotic episode can be 
treated with a number of other medication classes in addition to antipsychotics (e.g. antidepressants, mood stabilizers). It is reported that from around 16\% (up to about one third) of those with FEP will not have another episode and might show good long-term recovery. Around one third (up to 43\%) will have repeated episodes after FEP and an unremitted course of illness with increased impairment, while the rest will have multiple episodes over their lifetimes with partial or full remissions between episodes and varying patterns (Shepherd, Watt, Falloon, \& Smeeton, 1989; Messias, Chen, \& Eaton, 2007). We currently have no valid instrument to help us identify which FEP patient will fall into which category, and as mentioned previously, even our diagnoses depend on long-term observations of the clinical course.

Two points need to be emphasized and follow from what was presented about FEP. First, because of the progressive and deteriorating nature of "chronic" psychotic disorders (specifically those from the schizophrenia spectrum), it is vital to promote the early detection of psychosis and implement early interventions, and one of the ways to do that is to identify usable markers that might be shown to even precede the onset of manifest psychotic symptoms. Secondly, given the differences in the symptoms we see and the wide variety of possible outcomes (in terms of remission and level of functioning, but also clinical presentation changes), it is important to identify different predictors that might help us tailor interventions and individualize our approach to individuals with FEP. Both these markers and predictors could prove to be useful tools in our attempts to finally elucidate processes that underlie heterogeneous disorders like SCZ, their subtypes, and symptoms.

\section{Lexical-semantic deficits in schizophrenia}

As language disturbances are thought to be one of the most prominent characteristics of psychosis, and hence schizophrenia, from the beginning of their defining, they maintain the position of an important diagnostic criterion. Abnormalities of language production, such as derailment, tangentiality, poverty of speech, illogicality or incoherence, are commonly understood in terms of formal thought disorder (FTD) (Barrera et al., 2005, Kircher et al., 2018). FTD refers to a clinical description of a range of language production deficits which can be described as inadequacy of language production and alterations in the thought process (Kircheret al., 2018). The symptoms of FTD are most often categorized as being either positive or negative. Positive symptoms involve an increased amount of produced speech, including the production of neologisms, idiosyncratic associations, etc., while negative symptoms include deficits in speech and thought production. FTD occurs in a range of neuropsychiatric disorders, but 
chronic positive FTD is held as a diagnostic marker for schizophrenia and an indicator for high-risk psychosis (Kircher et al., 2018).

FTD is observed through traditional clinical scales and encompasses a variety of symptoms. Besides the aforementioned division into positive and negative symptoms, other categorizations include subjective vs. objective and qualitative vs. quantitative forms of the disorder. As these subdivisions present a heterogeneity of symptoms, different manifestations of the syndrome could represent different aspects of cognitive dysfunctions (Cavelti et al., 2018), and overall FTD diagnostics could obscure correlations between specific symptoms (Sumner et al., 2018). Lewandowski et al. (2018) remark that there is cognitive heterogeneity both within and between diagnostic categories, since patients across diagnoses share similar cognitive deficits, and patients with shared clinical and diagnostic features may exhibit different cognitive abnormalities. This heterogeneity presents problems for the study of cognitive dysfunctions in psychosis and should be further distinguished, with novel strategies and different approaches. Furthermore, these differences accentuate the importance of studies of first-episode and early-course psychosis along with longitudinal studies, as they could further enlighten the heterogeneity of symptoms accompanied with different cognitive or language deficits. First-episode and early-onset diagnoses, along with language and cognitive studies, could, if accompanied by longitudinal studies, distinguish subgroups of this heterogenic diagnosis which could reveal underlying processes in schizophrenia, and psychosis in general, and could become a valuable tool as a predictor for a particular course of the illness and its phases.

Brown and Kuperberg (2015) list four ways in which language disturbances are important in schizophrenia: language abnormalities manifest in symptoms of psychosis itself (particularly in positive FTD and auditory verbal hallucinations); language dysfunction is a core consequence of schizophrenia and is more strongly compromised in relation to other cognitive abilities; language production deficits often extend to perceptual processing of speech, i.e. comprehension; and language dysfunctions have been linked to functional and social impairments. Cavelti et al. (2018, citing Kircher, 2008; Strik et al., 2008) conclude that disturbances in the connectivity of the language network may be the underlying neurobiological cause of FTD language deficits. Hence, language deficits in schizophrenia may be tied exclusively to the semantic memory or may include other mechanisms of language processing. They may be due to a combination of deficits, the consequence of either a general deficit or a specific deficit.

The semantic memory is a long-term memory system containing organized knowledge about words and their meanings, referents, and relations (Tulving, 1972). It is considered to be organized into a network of associated concepts represented by nodes of interconnected links which indicate semantic relationships between them (Minzenberg, 2002, Collins and Loftus, 1975). Language process- 
ing deficits in schizophrenia may be tied exclusively to the semantic memory, but they also might include search and retrieval processes connected with executive functions and working memory processes. Although it is yet unclear which domains interact with which processes, both play a significant role in manipulating semantic memory concepts in language processing, in goal-directed processing, cognitive inhibition, flexibility, planning, etc. (Orellana and Slachevsky, 2013).

Studies have shown that, in schizophrenia, the semantic memory is preserved, meaning that the concepts and their features are not degraded as in category-specific deficits, but that access to them is disrupted (Allen et al., 1993, Elvevåg et al., 2002).

In 1938, Cameron (McKenna \& Oh, 2005) described an overinclusion of objects in categories by schizophrenia patients as compared to healthy controls. Lawrence et al. (2007) presented similar results, concluding that overinclusion is the result of an inability to preserve boundaries of categories, which leads to indistinct and broadened category boundaries. Other studies have reached similar results (Chen et al., 1994; Brébion et al., 2004; 2010; Kreher et al., 2008), while Elvevåg et al. (2002) did not find qualitative differences between schizophrenia patients and a healthy control group, but they did find slower reaction times in the patient group.

On account of hyperpriming results on priming tasks in schizophrenia patients, Spitzer (1993) concluded that patients have an increased amount of spreading activation in the semantic memory. In explaining hyperpriming in schizophrenia patients, Maher (1983) claimed that activating a concept activates concepts associated with the first activated concept, which in its foundation constitutes the theory of spreading activation. The increased spreading activation theory was validated with other studies, in addition to priming tests, by analyzing association production in schizophrenia patients' discourse (Maher et al., 2005), fluency tests (Johnson and Shean, 1993), categorization tests (Brébion et al., 2010, Kreher et al., 2008), etc.

Maher (1983) proposed that, along with the increase in spreading activation, inhibition processes in language processing are decreased in schizophrenia, resulting in the intrusion of activated, and not inhibited, associations (Leeson et al., 2005). In an ERP priming study, Kreher et al. (2008) also concluded that their results of heightened activation in a shorter time frame in the semantic memory of schizophrenia subjects, in comparison to healthy control subjects, could be the result of increased automatic activation and/or a deficiency of inhibition. Moritz et al. (2001), in a study on the processing of dominant and inferior meanings of homographs, also conclude that there is a lack of inhibition in schizophrenia patients compared to healthy control subjects.

Leeson et al. (2005) note that inhibition mechanisms play a significant role in both the increased spreading activation and disorganization theories. While, according to the spreading activation theory, inhibition seems to decrease in 
schizophrenia, resulting in heightened activation of the semantic memory and the activation of inappropriate concepts which would have been inhibited, the disorganized semantic memory theory suggests that inhibition is heightened as closely related activated concepts are inhibited and activation spreads to more distantly related concepts. Furthermore, inhibition is not the only executive function domain which seems to be affected in schizophrenia. Although abnormalities in both the semantic memory and the working memory or executive function have shown to predict clinical language disturbances (Kuperberg 2010), conclusions on their specific roles in language processing deficits in schizophrenia require further, more exhaustive studies.

On account of these theoretical approaches, Kuperberg et al. (2010) concluded that schizophrenia patients rely on semantic associations in language processing, which leads to the disruption of the balance of necessary processes and the generation of inappropriate meanings on a higher level. With further studies, Kuperberg et al. (2019) diverged research on schizophrenia language deficits towards controlled and automatic processes and top-down and bottom-up processing. Because patients with schizophrenia do not show heightened direct priming results, and on account of neural studies and highly automatic priming studies with 0 SOA, Kuperberg et al. (2019) concluded that increased automatic (indirect) priming occurs because of noisier lexical representations, i.e. nodes that describe the connections between the form and the meaning of words. While automatic spreading activation theory does not account for why schizophrenia patients mostly do not show heightened direct priming, Kuperberget al. (2019) suggest that automatic activity can spread in a bottom-up direct pathway from a prime word form representation to indirectly related representations, without them being meditated by a directly related representation, because the lexical representation encodes mappings between the word form and the semantic features (which do not need to be pre-activated) and are connected to indirectly related representations, hence "noisier lexical representations." These assumptions are based on the fact that, under automatic conditions at $0 \mathrm{SOA}$, it is suspected that there will not be enough time for pre-activation on the semantic level.

In a meta-analysis of studies concerning thought-disordered subjects, Sumner et al. (2018) conclude that there are few studies which investigate correlations between behavioral and functional neuroimaging measures in thought disorder, although there is behavioral evidence for executive dysfunctions in thought disorder. Even though neuroimaging studies mostly concern the analysis of a general symptom of attention and working memory, some neural correlates of thought disorder can be interpreted as executive function deficits, such as attentional selection (Pessoa, 2009) or cognitive integration (Duff and Brown-Schmidt, 2012). Accordingly, some cognitive evidence is indicative of combinatory semantic and executive dysfunctions in thought disorder (Leeson et al., 2005), as mentioned above. 


\section{The psycholinguistic characteristics of words in the assessment of language processing}

Any use of language (both in production and in perception), whether in healthy individuals or in individuals with some form of linguistic difficulty, necessarily involves access to information about the world that is stored in the form of lexical concepts and categories in the human mind. People shape mental concepts of categories of objects that enable them to respond appropriately to new objects they encounter. Concepts, their categorical organization, levels of hierarchical taxonomy, and ways of storing and retrieving information from lexical memory are the most commonly investigated psychological and neurolinguistic phenomena, especially in linguistic pathology (Levelt, 1993; Murphy, 2002; Murphy, 2010; Levin \& Pinker, 1992). The understanding and description of the mechanisms of language processing are primarily associated with semantic memory. Semantic memory is the term for long-term memory in which conceptual information (both semantic and lexical), as well as facts about the world, are presented. It refers to the part of long-term memory that processes ideas and concepts that do not derive from personal experience (as opposed to episodic memory) but which includes generally known facts acquired during one's life. This depository of signs, i.e. concepts or words and their characteristics, is thus found within the long-term memory, specifically within the semantic part of the declarative memory. Semantic memory derives from episodic memory inasmuch as new facts or concepts are learned based on experiences, while episodic memory is considered to intensify semantic memory. Semantic memory can also be defined as "a mental thesaurus, organized knowledge a person possesses about words and other verbal symbols, their meaning and referents, about relations among them, and about rules, formulas, and algorithms for the manipulation of these symbols, concepts, and relations" (Tulving, 1972, p. 386).

In order to understand the process of retrieving information from semantic memory in the language processing of persons with psychosis, ultra-high risk of psychosis (UHR), or schizophrenia, verbal fluency is used as a clinical test and as a basic tool. Verbal fluency can be semantic (the meaning relations of words within a category) and phonological (the connection between a word and its initial phoneme). Semantic verbal fluency is identified as a possible predictor of psychosis, neurocognitive deviations, and abnormalities and irregularities prevalent both in first-episode psychosis and in UHR patients. On the one hand, difficulties in semantic fluency may indicate problems in the very organization and storage of semantic information in the mental lexicon (category organization, levels of hierarchical taxonomy), but they may also indicate problems with the retrieval of information from lexical memory. Studies of UHR patients indicate impairment in selection, i.e. problems with selecting among alternatives during 
language processing, but not problems with retrieval from semantic memory. UHR patients have a lower mean value of total semantic fluency than help seeker controls. In young UHR patients, this effect is significant for each individual semantic category (e.g. animals, fruit), but not for phonological fluency. In patients with schizophrenia, semantic verbal fluency is also more impaired than phonological fluency. Difficult (poor) verbal fluency in schizophrenia may be an expression two separate problems in semantic processing - either the semantic memory has been damaged, or there is inefficient access to the semantic memory, which is actually normal.

The results of research on people at high risk of psychosis and non-psychotic help-seeking young adults, which confirmed significant differences on semantic fluency tests, suggest that semantic deficiencies occur during the prodromal phase and precede clinical expression, i.e. the full onset of psychosis. The very fact that verbal fluency (semantic categories) is impaired in patients at high risk for psychosis can be used to improve the predictability of the illness's development in high-risk patients, or in the possible transition to psychosis.

Therefore, some cognitive impairment in schizophrenia can be detected before the onset of the illness itself and thus can help identify people with high risk for psychosis. Recently great efforts have been invested in the development of fine-grained procedures for evaluating the measurement of multiple cognitive processes underlying performance. However, it is still unclear what independent components are needed to describe in detail the linguistic processing involved in a task of fluency.

Our goal in this book is to offer some of the possible linguistic elements (lexical characteristics) that could serve to improve the diagnostic value of early detection and illness monitoring tests in people with high risk for psychosis, first-episode psychosis, and schizophrenia.

Lexical units may be tightly or loosely connected in the mental lexicon, but their place in the semantic network is determined by the very fact that they share some of the same characteristics with other lexical units or their representations. Numerous linguistic and extralinguistic factors affect the storage and processing of language facts during spoken use. On the lexical level of language use, three basic types of parameters are most commonly said to be responsible for success in the use of words (Desrochers and Thompson, 2009; Desrochers et al., 2010). The first group consists of intrinsic characteristics of words that can be determined directly from their surface structure (e.g. the length of the words expressed in the number of phonemes, phones, letters, or syllables; part of speech; morphological characteristics, etc.). In the second group are parameters that depend on the target word's relationship to the wider corpus, i.e. contextual characteristics (the size of the phonological neighbourhood of the word, the word's frequency of use in the text, etc.). The third group of variables that affect 
the speed and accuracy of retrieving words from the mental lexicon during their active and passive use is constituted by so-called psycholinguistic characteristics, those which depend on the personal experience of the language user (e.g., the concreteness, subjective frequency, familiarity, and imageability of the word, the user's upon acquiring the word, etc.) and are calculated on the basis of the subjective assessment of a speakers of some language. We believe that precisely the psychological characteristics of the word could be a new element that would enable us to improve the diagnostic value of early detection and illness monitoring tests in people with high risk for psychosis, first-episode psychosis, and schizophrenia. Furthermore, we will address in particular those lexical characteristics that have been examined in some of the experimental studies presented in this book as well as characteristics that would be worth including in future studies.

\subsection{Imageability and concreteness}

Imageability is a psychological variable that addresses the ease at which a respondent creates a visual or auditory image of a reference that corresponds to a word; in other words, it is a lexical parameter that indicates how well a word leads to a mental image or sensory experience (Erdeljac, 2009; Erdeljac et al., 2014). The value of this characteristic is determined along a scale, so that a higher or lower degree of conceivability is attributed to individual concepts, and it is placed on the semantic level of language processing (Erdeljac et al., 2014). Imageability is commonly used in evaluating the effect of meaning on memory and word recognition. Imageability is often closely linked to concreteness, even to the extent that some authors either do not distinguish between these two lexical measures or they consciously equate them (Tyler and Moss, 1997; Paivio, 2010). But although for many words these parameters are in high correlation, there are data on subjective estimates that describe the same word as highly concrete while having low imageability. There are numerous studies which, taking into account this fact, prove that imageability is often a better predictor of the process of language performance than concreteness.

The key differences between the lexical-semantic systems of abstractness and concreteness consist in the fact that concrete words are adopted through sensory experience with physical objects, while abstract concepts are adopted through their use in sentences and in their mutual relations with other concepts within the language (Noppeney \& Price, 2004). With concrete concepts, the representational form varies depending on the modality in which it is represented (in visual, auditory, tactile, or gustatory form), while abstract concepts are formed $\mathrm{u}$ propozicijski. In addition, concrete concepts present a direct link with entities in the physical world and have a fixed frame of meaning, while abstract concepts 
are largely determined linguistic context. In accordance with these assumptions, concreteness is associated with perceptual characteristics, and abstractness with verbally learned information. People with deviations in language processing perform linguistic tasks more successfully with words that refer to concrete concepts than to those which refer to abstract concepts - they retrieve them more quickly and accurately from lexical memory (the mental lexicon), associate them more easily with other similar words, more successfully detect different types of semantic relations among them, and remember their meanings longer. Both concrete and abstract words are represented in the verbal system, while only concrete words are semantically coded in the non-verbal system (as they correspond to easily imageable representations). Precisely because representations of concrete words are in both systems, such words are easier to retrieve and are more accessible in the lexical memory (Erdeljac et al., 2014, pp. 41-42). Thus, abstract and concrete words are encoded differently in our mind: concrete words have both verbal and visual representations, while abstract words are only verbal/linguistic. While functionally separate, there is also a certain level of interconnectedness between the two systems, so that the activation of a representation in one system can activate the appropriate representation in the other. Concrete/Imageable words are more accessible precisely because the systems are connected, and both can be activated simultaneously (Tyler et al. 2002:476).

\subsection{Subjective frequency, age of acquisition, and familiarity of words}

Subjective frequency refers to how often, according to the subjective assessment of native speakers of a given language, a word is used or heard in daily communication. The frequency of words can be evaluated in two ways: objectively or subjectively. The objective frequency of a word refers to the sum of the occurrences of a word in text corpora, while the subjective frequency of a word is the result of the assessment of native speakers on a Likert scale (Desrochers \& Thompson, 2009).

A comparison of objective and subjective frequency norms showed a high correlation, so both modalities of assessment can be regarded as powerful predictors of ease and speed of responses in different types of language tasks (Fernández-Fuertes \& Thompson, 2010). In research on the processing of written (visual) and spoken (auditory) words, subjective frequency is a better predictor than objective frequency (Balota et al., 2001). Furthermore, the influence of lexical frequency on the speed of naming is confirmed, and words that are used or heard more often are easier to retrieve from lexical memory than low-frequency words. The frequency effect is undoubtedly situated at the level of phonological coding because it influences the time it takes to activate and select the target lexeme; this effect can be explained by lowering the threshold for the activation of 
the lexeme, which is low for frequent words and high for low-frequency words (Jescheniak \& Levelt, 1994).

Word frequency and age of acquisition are mutually very intertwined variables. The age of acquiring a word is defined as the period during which a person was able to understand a particular word if someone used it, even if he or she did not know how to use, read, or write that word at that time. It can be assumed that words adopted at an earlier/younger age tend to be used more often in adulthood by that speaker, but also that precisely the high frequency words (those used daily) are actually acquired earlier. These variables, it has been shown, are so mutually dependent that it is not easy to separate their effects. Age of acquisition has long been a neglected variable in lexical processing research, and its influences have been attributed to the frequency of words, which is easier to prove experimentally and to objectively observe (Barry et al., 2001).

Studies designed to observe the effects of word frequency and age of acquisition demonstrated the justification of the separation of these two characteristics of words, as it was confirmed that these two factors have different effects on the latency of response in different visual word processing tasks. It has been confirmed, for example, that age of acquisition affects naming latency, while word frequency is not confirmed in such types of tasks (Cortese \& Schock, 2012; Wilson, Cuetos, Davies, \& Burani, 2013).

Therefore, the subjective word frequency, as well as age of acquisition, are important variables that influence the processing of words. The greater frequency of a word and earlier acquisition of the word enable the speaker to retrieve and produce them more quickly. The processing of high-frequency words in tasks involving naming, as well as lexical and semantic decision-making, is faster than the processing of low-frequency words. It has been shown that frequency also plays a role in memorizing tasks such that low-frequency words are more difficult to invoke subsequently, but also that they lead to better results in tasks involving subsequent recognition. Subjective word frequency is a variable that correlates highly with many other characteristics - except with age of acquisition, it is also linked to word length, similarity to other words, etc.

The characteristics of word frequency and age of acquisition are also closely linked to knowledge of the word (the characteristic of familiarity). Familiarity refers to how often some object or concept is present in the experience of a speaker of a language. The familiarity of a word, or rather, of a concept, has been shown to have a significant impact on the speed of naming in healthy individuals as well as in those with some form of linguistic difficulty (aphasic patients). Images representing familiar objects retrieve the appropriate words from lexical memory more quickly than those representing unusual objects (Cuetos et al., 1999). Although this effect has not been found consistently in all studies investigating the determinants of image naming, in the literature, naming latency is 
considered an important possible linguistic processing predictor in pathology and should therefore be taken into account when this type of research is conducted (Alario et al., 2004; Barry et al., 1997).

It is assumed that familiarity affects the ease and speed of identifying objects and that this variable has an effect at the level of the link between visual recognition and the conceptual level (Cuetos et al., 1999). The effect of familiarity has been discovered at different levels of semantic processing in patients suffering from the semantic variant of primary progressive aphasia (Hirsh \& Funnell, 1995). The assumption is that the structural visual representations of very familiar objects activate their corresponding semantic representations than is the case with representations of objects that are not well known (Boukadi et al., 2016).

\subsection{Associative norms}

Associations are considered a reflection of semantic memorization because they provide insight into the internal structure of concepts in language processing. Within the framework of psychology and linguistics, studies of associations were introduced as part of research on memory and language processing in connection with various pathologies. Associations reflect the way in which human knowledge is structured, the semantic representations of words, and the relationships among them (de Groot, 1989). Free-association tests (the listing

of associations with a stimulus without further limitation, except limiting the quantity of data) are a means by which it is possible to construct maps of lexical knowledge of people who share linguistic and cultural background because similar experiences create associative structures that include representations of words and their connections, which are equated (Steyvers \& Tenenbaum 2005).

Associations can be based either on the meaning or the form of the word, but also on simultaneous similarity on both of these levels of representation (semantic and phonological-acoustic). The characteristics of associative links can be twofold, i.e. they can be unlimited in number and an unspecified in order, so that a specific linguistic sign, word, or concept can activate an undetermined number of associations in any order. On the one hand, one can talk about socalled clang associations, which include an associative link between words on an acoustic-phonological basis (rhyme, number of syllables, accents, etc.), and on the other hand, these may be paradigmatic associations, which link words of great semantic similarity. Semantic similarity may relate to the closeness or overlap of certain characteristics, to the occurrence of another word, or interchangeability, while syntagmatic associations are those between words that are often appear together.

In studies on the structure and organization of semantic memory, associations provide information about the associative structures that reflect representations 
of words, and the testing of associations has been carried out since the very beginnings of interest in semantic memory (even Tulving concluded that tests of the association provide wide insight into semantic memory, 1972, p. 402).

Associative norms provide insight into the relative accessibility of (associatively) related words in the semantic memory and enable insight into the strength of the connections between certain words in the semantic memory. Such norms are useful in linguistic research: in the construction of a network of semantic memory, they reveal the structure of freely remembering in learning and in language processing; they reveal methods of searching within different semantic domains; they serve as a standard for comparison in psychological studies of healthy populations and those with linguistic pathologies; they can be used as a base for creating language materials or a foundation for manipulation in tasks with different variables of characteristics.

Memory search is considered an automated process based on the characteristics of stimuli and is carried out according to the laws of association. There are many different psycholinguistic models that attempt to describe semantic processing. In connectivist models, the semantic memory is presented as a network of connections that represent the characteristics of concepts, while concepts are marked at the nodes of those connections, and activation spreads when the searching towards these links reaches nodes that represent concepts. The internal organization of the semantic storage is determined by the abstract links between concepts and does not depend on the modality of the concepts or their characteristics. Another applicable model for the description of semantic processing is the dual code theory (Paivio, 1986), which envisages two repositories of different modalities (verbal and visual storage) and a different activation that depends on modality. In this model, information is activated from one or both of the repositories. With respect to internal semantic organization, the models of the organization are distinguished by domain-specific categorization, according to which the concepts, depending on their category, are processed in different regions partly according to their sensory modalities. Spreading activation theory, upon which most of the studies presented in this book are based, is unimodal: it does not presuppose specific repositories for certain modalities and argues that complex knowledge located in the semantic memory consists of nodes and connections between nodes, and that the knowledge of one concept consists of a whole network of nodes connecting the network via connections. In the spreading of activation, or in semantic processing, inhibition plays a special role. 


\section{References}

Alario, X. F., Ferrand, L., Laganaro, M., New, B., Frauenfelder, U. H., \& Segui, J. (2004). Predictors of picture naming speed. Behavior Research Methods, Instruments, \& Computers, 36(1), 140-155.

Allen, H., Liddle, P. F., \& Frith, C. D. (1993, December). Negative features, retrieval processes and verbal fluency in schizophrenia. The British Journal of Psychiatry, 163(6), 769-775.

American Psychiatric Association. (2013). Diagnostic and statistical manual of mental disorders (5th ed.). Washington, DC: American Psychiatric Association.

Andreasen, N. C., Paradiso, S., \& Oleary, D. S. (1998). "Cognitive dysmetria" as an integrative theory of schizophrenia: A dysfunction in cortical-subcortical-cerebellar circuitry? Schizophrenia Bulletin, 24(2), 203-218. DOI:10.1093/oxfordjournals.schbul. a033321.

Balota, D. A., Pilotti, M., \& Cortese, M. J. (2001). Subjective frequency estimates for 2,938 monosyllabic words. Memory \& Cognition, 29, 639-647.

Barrera A., McKenna P. J., \& Berrios G. E. (2005). Formal thought disorder in schizophrenia: An executive or a semantic deficit? Psychological Medicine, 35(1), 121-32.

Barry, C., Hirsh, K. W., Johnston, R. A., \& Williams, C. L. (2001). Age of acquisitio$\mathrm{n}$,word frequency, and the locus of repetition priming of picture naming. Journal of Memory and Language, 44, 350-375.

Barry, C., Morrison, C. M., \& Ellis, A. W. (1997). Naming the Snodgrass and Vanderwart pictures: Effects of age of acquisition, frequency and name agreement. Quarterly Journal of Experimental Psychology, 50A, 560-585.

Bhugra, D. (2005). The global prevalence of schizophrenia. PLOS Medicine, 2(5): e151. https://doi.org/10.1371/journal.pmed.0020151

Boukadi, M., Zouaidi, C., \& Wilson M. A. (2016). Norms for name agreement, familiarity, subjective frequency, and imageability for 348 object names in Tunisian Arabic. Behavior Research Methods, 48(2), 585-599.

Brébion, G., Bressan, R. A., Ohlsen, R. I., Pilowsky, L. S., \& David, A. S. (2010). Production of atypical category exemplars in patients with schizophrenia. Journal of the International Neuropsychological Society, 16(5), 822-828

Brébion, G., David, A. S., Jones, H., \& Pilowsky, L. S. (2004). Semantic organization and verbal memory efficiency in patients with schizophrenia. Neuropsychology, 18(2), 378-383.

Breitborde, N. J., Srihari, V. H., \& Woods, S. W. (2009). Review of the operational definition for first-episode psychosis. Early Intervention in Psychiatry, 3(4), 259-265. https://doi.org/10.1111/j.1751-7893.2009.00148.x

Brewer, W. J., Wood, S. J., Phillips, L. J., Francey, S. M., Pantelis, C., Yung, A. R., Cornblatt, B., \& Mcgorry, P. D. (2005). Generalized and specific cognitive performance in clinical high-risk cohorts: A review highlighting potential vulnerability markers for psychosis. Schizophrenia Bulletin, 32(3), 538-555. https://doi.org/10.1093/ schbul/sbj077 
Brown, M., \& Kuperberg, G. R. (2015). A hierarchical generative framework of language processing: Linking language perception, interpretation, and production abnormalities in schizophrenia. Frontiers in Human Neuroscience, 9, 1-23.

Cavelti, M., Kircher, T., Nagels, A., Strik, W., \& Homan, P. (2018, September). Is formal thought disorder in schizophrenia related to structural and functional aberrations in the language network? A systematic review of neuroimaging findings. Schizophrenia Research, 199, 2-16, https://doi.org/10.1016/j.schres.2018.02.051

Chen, E. Y. H., Wilkins, A. J., \& McKenna, P. (1994, February). Semantic memory is both impaired and anomalous in schizophrenia. Psychological Medicine, 24(1), 193-202.

Collins, A. M., \& Loftus, A.S. (1975). A spreading activation theory of semantic processing. Psychological Review, 82, 407-428.

Cortese, M. J., \& Schock, J. (2012). Imageability and age of acquisition effects in disyllabic word recognition. Quarterly Journal of Experimental Psychology, 66(5), 946-972. https://doi.org/10.1080\%2F17470218.2012.722660

Cuetos, F., Ellis, A. W., \& Alvarez, B. (1999). Naming times for the Snodgrass and Vanderwart pictures in Spanish. Behavior Research Methods, Instruments and Computers, 31, 650-658.

De Groot, A. M. (1989). Representational aspects of word imageability and word frequency as assessed through word association. Journal of Experimental Psychology: Learning, Memory, and Cognition, 15(5), 824-845.

Desai, P. R., Lawson, K. A., Barner, J. C., \& Rascati, K. L. (2013). Estimating the direct and indirect costs for community-dwelling patients with schizophrenia. Journal of Pharmaceutical Health Services Research, 4(4), 187-194. https://doi.org/10.1111/jphs.12027

Desrochers, A., Liceras, J. M., Fernández-Fuertes, R., \& Thompson G. L. (2010). Subjective frequency norms for 330 Spanish simple and compound words. Behavior Research Methods, 42(1), 109-117.

Desrochers, A., \& Thompson, G. L. (2009). Subjective frequency and imageability ratings for 3,600 French nouns. Behavior Research Methods, 41(2), 546-557. https://doi. org/10.3758/BRM.41.2.546

Duff, M. C., \& Schmidt, S. B. (2012). The hippocampus and the flexible use and processing of language. Frontiers in Human Neuroscience, 6, Article ID 69.

Elvevåg, B., Weickert, T., Wechsler, M., Coppola, R., Weinberger, D. R., \& Goldberg, T. E. (2002). An investigation of the integrity of semantic boundaries in schizophrenia. Schizophrenia Research, 53, 187-198.

Erdeljac, V., Sekulić, M., Willer-Gold, J., Biočina, Z., Čolović, N., Dragojević, E., Feldman, E., Jelovac, T., Masnikosa, I., Rosandić, D. (2014) Leksičko obilježje predočivosti u mentalnom leksikonu osoba s afazijom. Govor 31(1), 29-47.

Erdeljac, V. (2009) Mentalni leksikon: modeli i činjenice, Ibis grafika d.o.o., Zagreb

Green, M. F., Kern, R. S., Braff, D. L., \& Mintz, J. (2000). Neurocognitive deficits and functional outcome in schizophrenia: Are we measuring the "right stuff"? Schizophrenia Bulletin, 26(1), 119-136. https://doi.org/10.1093/oxfordjournals.schbul.a033430

Häfner, H., Riecher-Rössler, A., Hambrecht, M., Maurer, K., Meissner, S., Schmidtke, A., Fätkenheuer, B., Löffler, W., \& Heiden, W. V. (1992). IRAOS: An instrument for the assessment of onset and early course of schizophrenia. Schizophrenia Research, 6(3), 209-223. https://doi.org/10.1016/0920-9964(92)90004-o 
Hilker, R., Helenius, D., Fagerlund, B., Skytthe, A., Christensen, K., Werge, T. M., Nordentoft, M., \& Glenthøj, B. (2018). Heritability of schizophrenia and schizophrenia spectrum based on the nationwide Danish twin register. Biological Psychiatry, 83(6), 492-498. https://doi.org/10.1016/j.biopsych.2017.08.017

Hirsh, K. W., \& Funnell, E. (1995). Those old, familiar things: Age of acquisition, familiarity and lexical access in progressive aphasia. Journal of Neurolinguistics, 9(1), 23-32. https://doi.org/10.1016/0911-6044(95)00003-8

Howes, O. D., \& Kapur, S. (2009). The dopamine hypothesis of schizophrenia: Version III--The final common pathway. Schizophrenia Bulletin, 35(3), 549-562. https://doi. org/10.1093/schbul/sbp006

Jescheniak, J. D., \& Levelt, W. J. M. (1994). Word frequency effects in speech production: Retrieval of syntactic information and of phonological form. Journal of Experimental Psychology: Learning, Memory, and Cognition, 20(4), 824-843.

Johnson, D. E., \& Shean, G. D. (1993). Word associations and schizophrenic symptoms. Journal of Psychiatric Research, 27(1). 69-77.

Kircher, T., Bröhl, H., Meier, F., \& Engelen J. (2018). Formal thought disorders: from phenomenology to neurobiology. Lancet Psychiatry 5(6) 515-26

Kreher, D. A., Holcomb, P. J., Goff, D., \& Kuperberg, G. R. (2008). Neural evidence for faster and further automatic spreading activation in schizophrenic thought disorder. Schizophrenia Bulletin 34(3), 473-482.

Kuperberg, G. R. (2010). Language in schizophrenia Part 1: An introduction. Language Linguist Compass 4(8), 576-589.

Kuperberg, G. R., Kreher, D. A., \& Ditman, T. (2010). What can Event-related Potentials tell us about language, and perhaps even thought, in schizophrenia? International Journal of Psychophysiology, 75, 66-76

Kuperberg, G. R., Weber K., Delaney-Busch, N., Ustine C., Stillerman B., Hämäläinen B., \& Lau E. (2019). Multimodal neuroimaging evidence for looser lexico-semantic networks in schizophrenia: Evidence from masked indirect semantic priming. Neuropsychologia 124, 337-349

Lawrence, V. A., Doughty, O., Al-Mousawi, A., Clegg, F., \& Done, D. J. (2007). Do overinclusion and distorted category boundaries in schizophrenia arise from executive dysfunction? Schizophrenia Research, 94, 172-179.

Leeson, V. C., Barnes, T. R., Hutton, S. B., Ron, M. A., \& Joyce, E. M. (2009). IQ as a predictor of functional outcome in schizophrenia: A longitudinal, four-year study of first-episode psychosis. Schizophrenia Research, 107(1), 55-60. https://doi.org/10.1016/j.schres.2008.08.014

Leeson, V. C., Simpson, A., McKenna, P. J., \& Laws, K. R. (2005). Executive inhibition and semantic association in schizophrenia. Schizophrenia Research, 74(1), 61-67.

Levelt, W. J. M. (1993). Speaking: From intention to articulation. Cambridge, MA: MIT Press.

Levin, B., \& Pinker, S. (Eds.). (1992). Lexical and conceptual semantics. Oxford: Blackwell.

Lewandowski K. E., McCarthy J. M., Öngür D., Norris L. A., Liu G. Z., Juelich R. J., \& Baker J. T. (2019). Functional connectivity in distinct cognitive subtypes in psychosis. Schizophrenia Research 204, 120-126. 
Maher, B.A. (1983). A tentative theory of schizophrenic utterance. In. B. A. Maher and W. B. Maher (Eds.), Progress in Experimental Personality Research, vol. 12: Personality (pp. 1-52). New York: Academic Press.

Maher, B. A., Manschreck, T. C., Linnet, J., \& Candela, S. 2005. Quantitative assessment of the frequency of normal associations in the utterances of schizophrenia patients and healthy controls. Schizophrenia Research 78(2-3), 219-224.

Mcgorry, P. D., Hickie, I. B., Yung, A. R., Pantelis, C., \& Jackson, H. J. (2006). Clinical staging of psychiatric disorders: A heuristic framework for choosing earlier, safer and more effective interventions. Australian and New Zealand Journal of Psychiatry, 40(8), 616-622. https://doi.org/10.1111/j.1440-1614.2006.01860

Mcgorry, P. D., Killackey, E., \& Yung, A. (2008). Early intervention in psychosis: Concepts, evidence and future directions. World Psychiatry, 7(3), 148-156. https://doi. org/10.1002/j.2051-5545.2008.tb00182

Mcgovern, D., \& Cope, R. V. (1987). First psychiatric admission rates of first and second generation Afro Caribbeans. Social Psychiatry, 22(3), 139-149. https://doi. org/10.1007/bf00583848

Mcgrath, J., Saha, S., Chant, D., \& Welham, J. (2008). Schizophrenia: A concise overview of incidence, prevalence, and mortality. Epidemiologic Reviews, 30(1), 67-76. https:// doi.org/10.1093/epirev/mxn001

McKenna, P., \& Oh, T. (2005). Schizophrenic speech: Making sense of bathroots and ponds that fall in doorways. New York, NY: Cambridge University Press.

Messias, E. L., Chen, C., \& Eaton, W. W. (2007). Epidemiology of schizophrenia: Review of findings and myths. Psychiatric Clinics of North America, 30(3), 323-338. https:// doi.org/10.1016/j.psc.2007.04.007

Minzenberg, M. J., Ober, B. A., \& Vinogradov, S. (2002). Semantic priming in schizophrenia: A review and synthesis. Journal of the International Neuropsychological Society, 8(5), 699-720.

Murphy, G. L. (2002). The Big Book of Concepts. Cambridge, MA: MIT Press, A Bradford book.

Murphy, G. L. (2010). What are categories and concepts? In D. Mareschal, P. C. Quinn, \& S. Lea (Eds.), The making of human concepts. Oxford: Oxford University Press.

Noppeney U., \& Price, C. J. (2004). Retrieval of abstract semantics, NeuroImage, 22(1), 164-170.

Nuechterlein, K. H., Barch, D. M., Gold, J. M., Goldberg, T. E., Green, M. F., \& Heaton, R. K. (2004). Identification of separable cognitive factors in schizophrenia. Schizophrenia Research, 72(1), 29-39. https://doi.org/10.1016/j.schres.2004.09.007

Orellana, G., \& Slachevsky, A. (2013). Executive functioning in schizophrenia. Frontiers in psychiatry, 4(35), 1-15.

Paivio, A. (1971). Imagery and verbal processes. New York: Holt, Rinehart \& Winston.

Paivio, A. (1986). Mental representations: A dual-coding approach. New York, Oxford University Press.

Paivio, A. (2010). Dual coding theory and the mental lexicon. The Mental Lexicon, 5(2), 205-230.

Paivio, A. \& Begg, I. (1981). The psychology of language. New York: Prentice-Hall. 
Pessoa, L. (2009). How do emotion and motivation direct executive control? Trends in Cognitive Sciences, 13, 160-166.

Sadock, B. J., Sadock, V. A., \& Ruiz, P. (2015). Synopsis of psychiatry: Behavioral sciences clinical psychiatry (11th ed.). New York: Wolters Kluwer.

Schizophrenia Working Group of the Psychiatric Genomics Consortium. (2014). Biological insights from 108 schizophrenia-associated genetic loci. Nature 511, 421-427. https://doi.org/10.1038/nature13595

Shepherd, M., Watt, D., Falloon, I., \& Smeeton, N. (1989). The natural history of schizophrenia: A five-year follow-up study of outcome and prediction in a representative sample of schizophrenics. Psychological Medicine Monograph Supplement, 15, 1-46. https://doi.org/10.1017/s026418010000059

Snodgrass, J. G., \& Yuditsky, T. (1996). Naming times for the Snodgrass and Vanderwart pictures. Behavior Research Methods, Instruments, \& Computers, 28(4), 516-536. https://doi.org/10.3758/BF03200540

Spitzer, M., Braun, U., Hermle, L., \& Maier, S. (1993). Associative semantic network dysfunction in thought-disordered schizophrenic patients: Direct evidence from indirect semantic priming. Biological Psychiatry, 34(12), 864-877.

Starc, M., Murray, J. D., Santamauro, N., Savic, A., Diehl, C., Cho, Y. T., Srihari, V., Morgan, P. T., Krystal, J. H., Wang, X. J., Repovs, G., \& Anticevic, A. (2017). Schizophrenia is associated with a pattern of spatial working memory deficits consistent with cortical disinhibition. Schizophrenia Research, 181, 107-116. https://doi. org/10.1016/j.schres.2016.10.011

Steyvers, M., \& Tenenbaum, J. B. (2005). The large-scale structure of semantic networks: Statistical analyses and a model of semantic growth. Cognitive Science 29(1), 41-78.

Sumner P. J., Bell I. H., \& Rossell S. R. 2018. A systematic review of the structural neuroimaging correlates of thought disorder. Neuroscience and Biobehavioral Reviews 84, 299-315.

Tulving, E. (1972). Episodic and semantic memory. In E. Tulving \& W. Donaldson (Eds.), Organization of memory (pp. 381-404). Oxford, England: Academic Press.

Tulving, E., \& Donaldson, W. (1972). Organization of memory. New York, London: Academic Press.

Tyler, L. K., de Mornay-Davies, P., Anokhina, R., Longworth, C., Randall, B., \& Marslen-Wilson, W. D. (2002). Dissociations in processing past tense morphology: Neuropathology and behavioral studies. Journal of Cognitive Neuroscience 14(1), 79-94.

Tyler, L., \& Moss, H. (1997) Functional properties of concepts: Studies of normal and brain damaged patients. Cognitive Neuropsychology, 14, 511-545.

Wilson, M. A., Cuetos, F., Davies, R., \& Burani, C. (2013). Revisiting age-of-acquisition effects in Spanish visual word recognition: The role of item imageability. Journal of Experimental Psychology: Learning, Memory, and Cognition. Advance online publication. https://doi.org/10.1037/a0033090

World Health Organization. (1992). The ICD-10 classification of mental and behavioural disorders: Clinical descriptions and diagnostic guidelines. Geneva: World Health Organization. 
Yang, G. J., Murray, J. D., Repovs, G., Cole, M. W., Savic, A., Glasser, M. F., Pittenger, C., Krystal, J. H., Wang, X. J., Pearlson, G. D., Glahn, D.C., \& Anticevic, A. (2014). Altered global brain signal in schizophrenia. Proceedings of the National Academy of Sciences, 111(20), 7438-7443. https://doi.org/10.1073/pnas.1405289111 